\title{
Plus-operators in Krein spaces and dichotomous behavior of irreversible dynamical systems with discrete time
}

\author{
by \\ V. Khatskevich (Karmiel) and L. Zelenko (Haifa)
}

\begin{abstract}
We study dichotomous behavior of solutions to a non-autonomous linear difference equation in a Hilbert space. The evolution operator of this equation is not continuously invertible and the corresponding unstable subspace is of infinite dimension in general. We formulate a condition ensuring the dichotomy in terms of a sequence of indefinite metrics in the Hilbert space. We also construct an example of a difference equation in which dichotomous behavior of solutions is not compatible with the signature of the indefinite metric.
\end{abstract}

1. Introduction. In $[\mathrm{Kh}-\mathrm{Z} 2]$ we considered a linear fractional transformation of the ball $\mathcal{K}^{-}$of all angular operators corresponding to the set of all maximal non-positive subspaces of a Krein space, i.e., of a Hilbert space $\mathcal{H}$ with an indefinite metric, for which both positive and negative components can be infinite-dimensional in general ([Kh2], [Kh3], [Az-I], [Kr]). This transformation is generated by a continuous linear operator $U$ in $\mathcal{H}$ (so-called bistrict plus-operator: see for example [Kh1]-[Kh3], [Az-I] and [Kr]). We did not assume $U$ to be continuously invertible. The weak compactness of the image and preimage of $\mathcal{K}^{-}$under the associated linear fractional transformation $F_{U}$ has been established ([Kh-Z2, Theorem 2.1]).

In the present paper we consider much more general operator linear fractional relations and we obtain some additional properties of the image and preimage of the ball $\mathcal{K}^{-}$(see Theorem 2.1, Proposition 2.1 and Example 3.1). We apply the above results to the study of dichotomous behavior of solutions to a non-autonomous linear difference equation in a Hilbert space $\mathcal{H}$,

$$
y_{n+1}=A_{n} y_{n} \quad\left(y_{n} \in \mathcal{H}, n=0,1,2, \ldots\right),
$$

2000 Mathematics Subject Classification: Primary 47B50, 37D99; Secondary 39A10, $47 \mathrm{~B} 37$.

Key words and phrases: linear difference equation in Hilbert space, plus-operator, linear fractional relation, dichotomous behavior.

The second author was supported by the KAMEA project for Scientific Absorption in Israel and in part by a grant from the German-Israeli Foundation (GIF). 
where $A_{n}$ are bounded linear operators acting in $\mathcal{H}$. This equation describes a non-autonomous dynamical system, in which the integer $n$ plays the role of discrete time. We do not assume that the operators $A_{n}$ are continuously invertible. This means that the corresponding dynamical system is irreversible in general. The dichotomous behavior means that solutions with initial values belonging to some subspace of the phase space $\mathcal{H}$ (the stable subspace) stabilize to zero at infinity, but all the other solutions grow indefinitely. For differential equations in Hilbert and Banach spaces this property was studied in [Mz], [Ms-Sh], [Kh-Z1], [Kh4], [Kh-Z2] and [Kh-Sh].

We formulate a condition ensuring dichotomous behavior in terms of the increment of a sequence $\left\{[\cdot, \cdot]_{n}\right\}_{n \in \mathbb{N}}$ of indefinite metrics with respect to equation (1.1) (see Definition 3.1 and Theorem 3.1) and apply the method of embedded bicones used in [Z], [Kh-Z1], [Kh4] and [Kh-Z2]. We obtain estimates for the velocity of the dichotomy. We pay a special attention to the case where the stable subspace is a maximal non-positive subspace associated with the indefinite metric $[\cdot, \cdot]_{n}$ for a large enough $n$ (see Theorem 3.1(iv)). In this case we say that the dichotomy is compatible with the signature of the indefinite metric. Close questions were considered and a similar approach was applied in [B-G1]-[B-G3]. In [B-G1] and [B-G3] the dichotomy of solutions of (1.1) was studied with the help of indefinite metrics in the case of a finite-dimensional Hilbert space $\mathcal{H}$. In particular, in [B-G3] the case of invertible operators $A_{n}$ was considered (a reversible system) and the method used was close to the method of embedded bicones (see the proof of Lemma 4.4 of [B-G3]). In [B-G2] the case of an infinite-dimensional Hilbert space was studied and the so-called inertia theorems for (1.1) were obtained (see Remark 3.1 of the present paper).

If the operators $A_{n}$ in (1.1) are not continuously invertible, application of the method of embedded bicones faces certain topological difficulties. To overcome these, we use Theorem 2.1 of [Kh-Z2] mentioned above. We also construct an example of a dichotomous dynamical system in which the dichotomy is not compatible with the signature of the indefinite metric (Example 3.2).

Notice that another approach to the dichotomy of the dynamical system (1.1) (see [B-G1], [B-G4], [B-G5] $\left({ }^{1}\right),[\mathrm{A}-\mathrm{M}-\mathrm{Z}],[\mathrm{A}-\mathrm{S}]$ and $[\mathrm{B}-\mathrm{P}]$ ) is to study the connection of the dichotomy with the Fredholm properties of the operator

$$
(L \mathbf{y})_{n}=y_{n+1}-A_{n} y_{n}
$$

(so-called "weighted shift"), acting in the Hilbert space $L_{2}\left(\mathbb{Z}_{+}, \mathcal{H}\right)$ of sequences $\mathbf{y}=\left\{y_{n}\right\}_{n \in \mathbb{Z}_{+}}\left(y_{n} \in \mathcal{H}\right)$. In particular, it turns out [B-G4] that the exponential dichotomy of a reversible dynamical system of the form (1.1)

( $\left.{ }^{1}\right)$ In [B-G1]-[B-G5] a more general version of equation (1.1) was considered. 
in a finite-dimensional Hilbert space $\mathcal{H}$ is equivalent to the continuous invertibility of the operator $L$ acting in $L_{2}(\mathbb{Z}, \mathcal{H}$ ) (after a suitable extension of $\left\{A_{n}\right\}_{n \in \mathbb{Z}_{+}}$to the negative time axis).

Now we describe the contents of the paper.

At the beginning of Section 2 we give the needed background from the theory of spaces with an indefinite metric. In particular, the concepts of plus-operator, strict plus-operator and bistrict plus-operator are defined. Moreover, we introduce a couple of new notions: a linear fractional relation $F_{U}$ (LFR for brevity), defined by the block-matrix of a plus-operator $U$, the dual notion of an LFR $G_{T}$ defined by a minus-operator $T$, and some operator sets $\mathcal{G}_{U}^{ \pm}$. These notions have a conceptual character and Theorem 2.1 is an evidence of this. It asserts that in the case of an invertible bistrict plusoperator $U$ the image $\operatorname{Im}\left(G_{T}\right)\left(T=U^{-1}\right)$ is exactly the set $\mathcal{G}_{U}^{-}$(equation (2.6)). And vice versa: if (2.6) holds with $T=U^{-1}$, then $U$ is a bistrict plus-operator (consequently, $T$ is a bistrict minus-operator, and $G_{T}$ is a usual linear fractional transformation, LFT for brevity). This fact defines the borders of the method used in [Kh-Sh], where the case of a reversible continuous time dynamical system was considered, that is, the evolution operator $U(t)$ was assumed to be invertible. In [Kh-Sh] the compactness of $\operatorname{Im}\left(G_{T}\right)\left(T=U^{-1}\right)$ in the weak operator topology (WOT) was established by using (2.6) and the fact that the image of an LFT is compact in WOT. This compactness is a key point of the proof of the dichotomy of solutions $x(t)=U(t) x_{0}$ to the evolution equation in [Kh-Sh]. In view of Theorem 2.1, we now see that in the general case of a plus-operator $U(t)$ (not necessarily bistrict as in [Kh-Z2] or invertible as in [Kh-Sh]) one has to develop a general theory of LFRs of types (2.3)-(2.4) (see also Remark 2.1). In [Kh4, Section 2] a number of results on geometrical and topological structure of images and domains of LFRs (called there generalized linear fractional transformations) were obtained. Our Theorem 2.1 is also a result of such type.

The final Proposition 2.1 of Section 2 asserts that the spectrum $\sigma(U)$ of a strict plus-operator $U$ such that $\mathcal{G}_{U}^{-}=\emptyset$ surrounds the origin of the complex plane $\mathbb{C}$. This result is closely related to a very interesting problem concerning existence of a continuous time dynamical system with the dichotomy of solutions which is not compatible with the signature of an indefinite metric (see details in Remark 3.4).

In Section 3 we prove the above mentioned Theorem 3.1 about dichotomy of solutions of irreversible dynamical systems with discrete time. Furthermore, we construct examples of dynamical systems with dichotomy of solutions incompatible with an indefinite metric (see Examples 3.1 and 3.2).

Let us introduce some notation:

- $\mathbb{C}$ is the set of all complex numbers; 
- $\mathbb{R}_{+}=[0, \infty)$;

- $\mathbb{N}$ is the set of all natural numbers;

- $\mathbb{Z}_{+}=\{0\} \cup \mathbb{N}$;

- $\mathbb{T}=\{z \in \mathbb{C}|| z \mid=1\}$;

- $\operatorname{cl}(\mathcal{S})$ is the closure of a subset $\mathcal{S}$ of a Banach space $\mathcal{B}$;

- $\mathcal{H}$ is a complex or real Hilbert space with inner product $(x, y)$ and norm $\|x\|=(x, x)^{1 / 2}$; we will also use $\mathcal{H}$ with subscripts, but they will be omitted in the notation of norms and inner products;

- $\operatorname{span}(\mathcal{S})$ is the minimal closed subspace of a Hilbert space $\mathcal{H}$ containing a subset $\mathcal{S}$ of $\mathcal{H}$;

- $\mathcal{L}\left(\mathcal{H}_{1}, \mathcal{H}_{2}\right)$ is the set of all bounded linear operators acting from a Hilbert space $\mathcal{H}_{1}$ to a Hilbert space $\mathcal{H}_{2}$; if $\mathcal{H}_{1}=\mathcal{H}_{2}=\mathcal{H}$, we will write $\mathcal{L}(\mathcal{H})$

- $\mathcal{S} \mathcal{L}(\mathcal{H})$ is the set of all selfadjoint operators from $\mathcal{L}(\mathcal{H})$;

- $\sigma(A)$ is the spectrum of a linear operator $A$ acting in $\mathcal{H}$;

- $(\mathcal{H}, V)$ is a Krein space, i.e. $\mathcal{H}$ is a Hilbert space with an indefinite metric $[x, y]=(V x, y)$, where $V \in \mathcal{S} \mathcal{L}(\mathcal{H})$ is continuously invertible;

- $\mathcal{H}^{+}, \mathcal{H}^{-}$(possibly with subscripts) are the invariant subspaces of $V$, corresponding to the positive and negative parts of its spectrum, respectively; $d_{V}^{+}=\operatorname{dim}\left(\mathcal{H}^{+}\right), d_{V}^{-}=\operatorname{dim}\left(\mathcal{H}^{-}\right)$(these numbers can be infinite in general);

- $\mathcal{P}^{+}, \mathcal{P}^{-}$(possibly with subscripts) are respectively the non-negative and non-positive bicones generated by the indefinite metric $[x, y]$ : $\mathcal{P}^{+}=\{x \in \mathcal{H}:[x, x] \geq 0\}, \mathcal{P}^{-}=\{x \in \mathcal{H}:[x, x] \leq 0\}$.

2. Plus-operators and linear fractional relations. Let $(\mathcal{H}, V)$ be a Krein space, $\mathcal{H}=\mathcal{H}^{+} \oplus \mathcal{H}^{-}$. Denote by $P^{+}, P^{-}$the orthogonal projections on $\mathcal{H}^{+}, \mathcal{H}^{-}$respectively. It is well known that one can reduce the regular indefinite square form $[x, x]=(V x, x)$ to the form

$$
[x, x]=\left\|P^{+} x\right\|^{2}-\left\|P^{-} x\right\|^{2},
$$

by passing to an equivalent norm in $\mathcal{H}[\mathrm{Az}-\mathrm{I}]$. In the following we will assume such passage to be realized. Recall that a subspace $L \subseteq \mathcal{H}$ is called non-positive (respectively, non-negative) if it is contained in $\mathcal{P}^{-}$(respectively, in $\mathcal{P}^{+}$). A non-positive (respectively, non-negative) subspace $L \subseteq \mathcal{H}$ is called maximal non-positive (respectively, maximal non-negative) if it is not a proper subset of any non-positive (respectively, non-negative) subspace.

Consider two Krein spaces $\left(\mathcal{H}_{i}, V_{i}\right)(i=1,2)$ with indefinite metrics of the form defined above, i.e. $V_{i}=P_{i}^{+}-P_{i}^{-}$, where $P_{i}^{+}, P_{i}^{-}$are the orthogonal projections in $\mathcal{H}_{i}$ on the subspaces $\mathcal{H}_{i}^{+}, \mathcal{H}_{i}^{-}$respectively. This means that we have orthogonal splittings

$$
\mathcal{H}_{i}=\mathcal{H}_{i}^{+} \oplus \mathcal{H}_{i}^{-}, \quad i=1,2 .
$$


Assume that the signatures of the quadratic forms $[x, x]_{i}=\left(V_{i} x, x\right)$ are the same, i.e. $d_{V_{1}}^{+}=d_{V_{2}}^{+}$and $d_{V_{1}}^{-}=d_{V_{2}}^{-}$. Let $\mathcal{P}_{i}^{+}, \mathcal{P}_{i}^{-}(i=1,2)$ be the non-negative and non-positive bicones respectively generated by the above indefinite metrics.

Recall that a bounded linear operator $U$ acting from $\left(\mathcal{H}_{1}, V_{1}\right)$ to $\left(\mathcal{H}_{2}, V_{2}\right)$ is called a plus-operator if $x \in \mathcal{P}_{1}^{+}$implies $U x \in \mathcal{P}_{2}^{+}$, and it is called a strict plus-operator if

$$
[U x, U x]_{2} \geq \mu(U)[x, x]_{1} \quad \forall x \in \mathcal{H}_{1},
$$

where $\mu(U)>0$. If together with $U$ the adjoint operator $U^{\star}$ is also a strict plus-operator, then $U$ is called a bistrict plus-operator. The definitions of a minus-operator (strict and bistrict) are analogous (see $[\mathrm{Az}-\mathrm{I}]$ ).

Consider the block matrix representation of the operator $U$, corresponding to the orthogonal decompositions (2.1):

$$
U=\left\{U_{i, j}\right\}_{i, j=1}^{2} \text {. }
$$

Let $\mathcal{M}_{i}^{+}$(resp., $\mathcal{M}_{i}^{-}$) be the set of all maximal non-negative (resp., maximal non-positive) subspaces of $\left(\mathcal{H}_{i}, V_{i}\right), i=1,2$. We denote by $\mathcal{K}_{i}^{+}$(resp., $\mathcal{K}_{i}^{-}$) the set of all angular operators of subspaces $L_{i}^{+} \in \mathcal{M}_{i}^{+}$(resp., $L_{i}^{-} \in \mathcal{M}_{i}^{-}$), $i=1,2$. Recall that these are the linear operators $K_{i}^{+}: \mathcal{H}_{i}^{+} \rightarrow \mathcal{H}_{i}^{-}$(resp., $\left.K_{i}^{-}: \mathcal{H}_{i}^{-} \rightarrow \mathcal{H}_{i}^{+}\right)$for which $L_{i}^{+}=\operatorname{graph}\left(K_{i}^{+}\right)\left(L_{i}^{-}=\operatorname{graph}\left(K_{i}^{-}\right)\right)$. Let us also recall $[\mathrm{Az}-\mathrm{I}]$ that any non-negative (resp., non-positive) subspace is contained in some maximal non-negative (resp., maximal non-positive) subspace. In other words, for any $E_{i}^{+} \subset \mathcal{P}_{i}^{+}$(resp., $E_{i}^{-} \subset \mathcal{P}_{i}^{-}$) there exists $K_{i}^{+} \in \mathcal{K}_{i}^{+}$(resp., $\left.K_{i}^{-} \in \mathcal{K}_{i}^{-}\right)$such that

$$
E_{i}^{+} \subset L_{i}^{+}=\left(P_{i}^{+}+K_{i}^{+}\right) \mathcal{H}_{i}^{+} \quad\left(\text { resp. }, E_{i}^{-} \subset L_{i}^{-}=\left(P_{i}^{-}+K_{i}^{-}\right) \mathcal{H}_{i}^{-}\right)
$$

(see details in $[\mathrm{Az}-\mathrm{I}],[\mathrm{Kh} 1])$. Define

$$
\begin{gathered}
\mathcal{G}_{U}^{-}=\left\{K_{1}^{-} \in \mathcal{K}_{1}^{-}: U\left(P_{1}^{-}+K_{1}^{-}\right) \mathcal{H}_{1}^{-} \subset \mathcal{P}_{2}^{-}\right\} \\
\mathcal{G}_{U}^{+}=\left\{K_{2}^{+} \in \mathcal{K}_{2}^{+}:\left(P_{2}^{+}+K_{2}^{+}\right) \mathcal{H}_{2}^{+} \supseteq U\left(P_{1}^{+}+K_{1}^{+}\right) \mathcal{H}_{1}^{+} \text {for some } K_{1}^{+} \in \mathcal{K}_{1}^{+}\right\} .
\end{gathered}
$$

For a plus-operator $U$ define a linear fractional relation (LFR for brevity) $F_{U}: \mathcal{K}_{1}^{+} \rightarrow \mathcal{K}_{2}^{+}$by

$$
F_{U}\left(K_{1}^{+}\right)=\left\{K_{2}^{+} \in \mathcal{K}_{2}^{+}: U_{2,1}+U_{2,2} K_{1}^{+}=K_{2}^{+}\left(U_{1,1}+U_{1,2} K_{1}^{+}\right)\right\} .
$$

In the particular case of a bistrict plus-operator $U$, the LFR $F_{U}$ becomes a usual single-valued linear fractional mapping (LFM for brevity) of the form

$$
F_{U}\left(K_{1}^{+}\right)=\left(U_{2,1}+U_{2,2} K_{1}^{+}\right)\left(U_{1,1}+U_{1,2} K_{1}^{+}\right)^{-1} .
$$

Analogously, for a minus-operator $T: \mathcal{H}_{2} \rightarrow \mathcal{H}_{1}$ define an LFR $G_{T}: \mathcal{K}_{2}^{-} \rightarrow$ $\mathcal{K}_{1}^{-}$as follows:

$$
G_{T}\left(K_{2}^{-}\right)=\left\{K_{1}^{-} \in \mathcal{K}_{1}^{-}: T_{1,1} K_{2}^{-}+T_{1,2}=K_{1}^{-}\left(T_{2,1} K_{2}^{-}+T_{2,2}\right)\right\} .
$$


Let us notice that $K_{1}^{-} \in G_{T}\left(K_{2}^{-}\right)$is equivalent to $T L_{2}^{-} \subseteq L_{1}^{-}$, where $L_{i}^{-}=\left(P_{i}^{-}+K_{i}^{-}\right) \mathcal{H}_{i}^{-} \in \mathcal{M}_{i}^{-}$. In the particular case when $T L_{2}^{-} \in \mathcal{M}_{i}^{-}$we have the equality $T L_{2}^{-}=L_{1}^{-}$. Hence in this case $G_{T}\left(K_{2}^{-}\right)$is a single point of $\mathcal{K}_{1}^{-}$and $K_{1}^{-}=G_{T}\left(K_{2}^{-}\right)$. In the general case $G_{T}\left(K_{2}^{-}\right)$is the set of all the operators $\widetilde{K}_{1}^{-} \in \mathcal{K}_{1}^{-}$such that the restriction $\left.\widetilde{K}_{1}^{-}\right|_{P_{1}^{-} T L_{2}^{-}}$coincides with $P_{1}^{+}\left(\left.P_{1}^{-}\right|_{T L_{2}^{-}}\right)^{-1}$ (see $\left.[\mathrm{Az}-\mathrm{I}]\right)$.

Consider some basic properties of LFRs. First note that it follows immediately from the definition that

$$
\mathcal{G}_{c U}^{+}=\mathcal{G}_{U}^{+}, \quad \mathcal{G}_{c U}^{-}=\mathcal{G}_{U}^{-}, \quad \text { for } 0 \neq c \in \mathbb{C} .
$$

Evidently, $\operatorname{Im}\left(F_{U}\right)=\mathcal{G}_{U}^{+}$. The following assertion establishes the relation between $\mathcal{G}_{U}^{-}$and $\operatorname{Im}\left(G_{U^{-1}}\right)$.

Theorem 2.1. Let $U$ be an invertible plus-operator. Then $T=U^{-1}$ is a minus-operator. Furthermore,

$$
\mathcal{G}_{U}^{-}=\operatorname{Im}\left(G_{T}\right)
$$

if and only if $U$ is a bistrict plus-operator.

Proof. Since $U$ is an invertible plus-operator, it follows that $T=U^{-1}$ is a minus-operator. As a consequence of invertibility of $U$ we see $[\mathrm{Az}-\mathrm{I}]$ that $U$ and $U^{-1}$ are respectively a strict plus-operator and a strict minus-operator. Now suppose in addition $U$ is bistrict, that is, both $U$ and $U^{\star}$ are strict plus-operators. Then ([Kh1], $[\mathrm{Az}-\mathrm{I}]) T$ is a bistrict minus-operator and

$$
T L_{2}^{-} \in \mathcal{M}_{1}^{-} \quad \forall L_{2}^{-} \in \mathcal{M}_{2}^{-} .
$$

Now let $K_{1}^{-} \in \operatorname{Im}\left(G_{T}\right)$ and $L_{1}^{-}=\left(P_{1}^{-}+K_{1}^{-}\right) \mathcal{H}_{1}^{-} \in \mathcal{M}_{1}^{-}$. Then by the definition of $\operatorname{Im}\left(G_{T}\right)$ there exists $K_{2}^{-} \in \mathcal{K}_{2}^{-}$such that $T L_{2}^{-} \subseteq L_{1}^{-}$, where $L_{2}^{-}=\left(P_{2}^{-}+K_{2}^{-}\right) \mathcal{H}_{2}^{-} \in \mathcal{M}_{2}^{-}$. Hence by $(2.7), T L_{2}^{-}=L_{1}^{-}$, and consequently, $U L_{1}^{-}=L_{2}^{-}$, that is, $K_{1}^{-} \in \mathcal{G}_{U}^{-}$.

Now let $K_{1}^{-} \in \mathcal{G}_{U}^{-}$and as above $L_{1}^{-}=\left(P_{1}^{-}+K_{1}^{-}\right) \mathcal{H}_{1}^{-}$. Then by $(2.7)$, $L_{2}^{-}=U L_{1}^{-} \in \mathcal{M}_{2}^{-}$, that is, there exists $K_{2}^{-} \in \mathcal{K}_{2}^{-}$such that $L_{2}^{-}=\left(P_{2}^{-}+\right.$ $\left.K_{2}^{-}\right) \mathcal{H}_{2}^{-}$. As a result we have $T L_{2}^{-}=L_{1}^{-}$, hence $K_{1}^{-} \in \operatorname{Im}\left(G_{T}\right)$. So, we have proved the equality (2.6).

Now assume that $\mathcal{G}_{U}^{-}=\operatorname{Im}\left(G_{T}\right)$. Our aim is to prove that $U$ is a bistrict plus-operator. As mentioned above, $U$ is strict. Take any $K_{1}^{-} \in \mathcal{G}_{U}^{-}$and set $L_{1}^{-}=\left(P_{1}^{-}+K_{1}^{-}\right) \mathcal{H}_{1}^{-}$. By the definition of $\mathcal{G}_{U}^{-}, \widetilde{L}_{2}^{-}=U L_{1}^{-}$is a nonpositive subspace, so it belongs to some maximal non-positive subspace $L_{2}^{-}$ with the angular operator $K_{2}^{-}: L_{2}^{-}=\left(P_{2}^{-}+K_{2}^{-}\right) \mathcal{H}_{2}^{-}$and $U L_{1}^{-} \subseteq L_{2}^{-}$. At the same time $K_{1}^{-} \in \operatorname{Im}\left(G_{T}\right)$ and $T=U^{-1}$. Hence $L_{1}^{-} \supseteq U^{-1} L_{3}^{-}$, where $L_{3}^{-}=\left(P_{2}^{-}+K_{3}^{-}\right) \mathcal{H}_{2}^{-}$for some $K_{3}^{-} \in \mathcal{K}_{2}^{-}$. As a result we obtain

$$
L_{3}^{-} \subseteq U L_{1}^{-} \subseteq L_{2}^{-},
$$


where both $L_{2}^{-}$and $L_{3}^{-}$are maximal non-positive subspaces (that is, $L_{2}^{-}, L_{3}^{-}$ $\left.\in \mathcal{M}_{2}^{-}\right)$. Consequently,

$$
U L_{1}^{-}=L_{2}^{-}=L_{3}^{-}, \quad U^{-1} L_{2}^{-}=U^{-1} L_{3}^{-}=L_{1}^{-} .
$$

This means that the strict minus-operator $U^{-1}$ takes the maximal nonpositive subspace $L_{2}^{-}$onto the maximal non-positive subspace $L_{1}^{-}$. Therefore $[\mathrm{Az}-\mathrm{I}], U^{-1}$ is a bistrict minus-operator and consequently $U$ is a bistrict plus-operator.

REMARK 2.1. In [Kh-Sh] a reversible dynamical system was considered with an invertible bistrict evolution plus-operator $U$, and dichotomy of solutions, compatible with the signature of the indefinite metric, was proved. The method used in [Kh-Sh] is based on replacing $\mathcal{G}_{U}^{-}$by $\operatorname{Im}\left(G_{U^{-1}}\right)$. Theorem 2.1 shows that even in the case of an invertible strict but not bistrict plus-operator $U$ the method of [Kh-Sh] cannot be used to prove the compatible dichotomy of solutions.

In $[\mathrm{Kh}-\mathrm{Z} 2]$ we proved the following

THEOREM 2.2. Let $U$ be a bounded bistrict plus-operator acting from $\left(\mathcal{H}_{1}, V_{1}\right)$ to $\left(\mathcal{H}_{2}, V_{2}\right)$. If $d_{V_{1}}^{-} \neq 0$ then the set $\mathcal{G}_{U}^{-}$is non-empty and compact with respect to the weak operator topology in $\mathcal{L}\left(\mathcal{H}_{1}^{-}, \mathcal{H}_{1}^{+}\right)$.

REMARK 2.2. In [Kh4] the compactness of $\mathcal{G}_{U}^{-}$with respect to the weak operator topology was also proved in the general case of an arbitrary bounded plus-operator $U$ (not bistrict and even not strict). But even in the case of a strict (but not bistrict) plus-operator $U$ the set $\mathcal{G}_{U}^{-}$can be empty in the non-trivial situation $d_{V_{1}}^{-} \neq 0$. The corresponding example was constructed in [Kh-Z2, Example 2.1] (see also Example 3.1 of the present paper).

Let us introduce the following

Definition 2.1. A plus-operator $U$ is called $J$-expansive if

$$
[U x, U x]_{2} \geq[x, x]_{1} \quad \forall x \in \mathcal{H}_{1},
$$

and $J$-biexpansive if both $U$ and $U^{\star}$ are $J$-expansive.

Let $U$ act in a Krein space $(\mathcal{H}, V)$, that is, $U: \mathcal{H} \rightarrow \mathcal{H}$. Then we have

Proposition 2.1. If $U$ is a strict plus-operator in $\mathcal{H}$ and

$$
\mathcal{G}_{U}^{-}=\emptyset
$$

then there exists a circle $C_{r}$ with center zero and radius $r>0$ such that $C_{r} \subseteq \sigma(U)$.

Proof. Define $\widetilde{U}=(\mu(U))^{-1 / 2} U$. Then $\widetilde{U}$ is a $J$-expansive operator: $[\widetilde{U} x, \widetilde{U} x] \geq[x, x]$ for all $x \in \mathcal{H}$. Assume that there is a complex number 
$\lambda$ with $|\lambda|=1$ such that $\lambda$ is a regular point of $\widetilde{U}$. Without loss of generality consider $\lambda=1$. Then $\widetilde{U}$ (see [Az-I, proof of Theorem II.4.31] $\left({ }^{2}\right)$ ) is a $J$-biexpansive operator, and consequently, $U$ is a bistrict plus-operator. Hence by [Kh-Z2] we conclude that $\mathcal{G}_{U}^{-} \neq \emptyset$.

REMARK 2.3. Notice that in the case $d_{V}^{-}=0$ the only maximal nonpositive subspace is $\mathcal{H}^{-}=\{0\}$. Each linear operator $U$ maps it into itself, so (2.8) never holds. On the other hand, any operator $U$ such that $\|U x\| \geq d\|x\|$ with $d>0$ is a strict plus-operator. That is, in this case $\sigma(U)$ may surround the origin for some strict plus-operators (for example, for the bilateral shift) and not surround it for others (for example, for the identity operator $U=I$ ).

3. Dichotomy of solutions to the evolution equation. We now turn to the linear difference equation of the form (1.1) in a Hilbert space $\mathcal{H}$. Recall that we have assumed in the Introduction that all the operators $A_{n}$ in this equation belong to the class $\mathcal{L}(\mathcal{H})$. Consider the evolution operator $U(n, m)\left(n, m \in \mathbb{Z}_{+}, n \geq m\right)$ of equation (1.1). Recall that it associates with each $y_{0} \in \mathcal{H}$ the solution $y_{n}$ of (1.1) satisfying the initial condition $\left.y_{n}\right|_{n=m}=y_{0}$, that is,

$$
U(n, m) y_{0}=A_{n-1} A_{n-2} \cdots A_{m+1} A_{m} y_{0} \quad \text { if } n>m,
$$

and $U(n, n)=I$. We shall write briefly $U(n)=U(n, 0)$. Along with (1.1) consider the following sequence of difference equations in $\mathcal{H}$ :

$$
z_{n+1}=A_{N-n}^{\star} z_{n},
$$

where $N \in \mathbb{Z}_{+}, n=0,1, \ldots, N$. For each fixed $N \in \mathbb{Z}_{+}$denote by $\widetilde{U}_{N}(n, m)$ the evolution operator of this equation. In view of (3.1), these operators are linked to the evolution operator of (1.1) by

$$
\widetilde{U}_{n+m-1}(n, m)=U^{\star}(n, m),
$$

hence for $m=0$,

$$
U^{\star}(n)=\widetilde{U}_{n-1}(n) .
$$

Consider a sequence of indefinite metrics in $\mathcal{H}$ of the form

$$
[x, y]_{n}=\left(V_{n} x, y\right) \quad\left(n \in \mathbb{Z}_{+}\right),
$$

where each operator $V_{n}$ belongs to $\mathcal{S L}(\mathcal{H})$, is continuously invertible and satisfies the following conditions:

(A) The numbers $d_{V_{n}}^{+}, d_{V_{n}}^{-}$do not depend on $n$.

(B) $\sup _{n \in \mathbb{Z}_{+}}\left\|V_{n}\right\|=1$.

Let us introduce the following

$\left({ }^{2}\right)$ The idea to use this theorem was suggested to us by T. Ya. Azizov. 
Definition 3.1. We call the sequence of quadratic forms

$$
\left[U(n+1, n) y_{0}, U(n+1, n) y_{0}\right]_{n+1}-\left[y_{0}, y_{0}\right]_{n} \quad\left(n \in \mathbb{Z}_{+}\right)
$$

the increment of the sequence of the quadratic forms $\left[y_{0}, y_{0}\right]_{n}$ with respect to the difference equation (1.1) and denote it by $\Delta_{(1.1)}\left(\left[y_{0}, y_{0}\right]_{n}\right)$.

From (3.4), (1.1) we obtain

$$
\begin{aligned}
\Delta_{(1.1)}\left(\left[y_{0}, y_{0}\right]_{n}\right) & =\left[A_{n} y_{0}, A_{n} y_{0}\right]_{n+1}-\left[y_{0}, y_{0}\right]_{n} \\
& =\left(\left(A_{n}^{\star} V_{n+1} A_{n}-V_{n}\right) y_{0}, y_{0}\right) .
\end{aligned}
$$

In the analogous manner we obtain

$$
\begin{aligned}
\Delta_{(3.2)}\left(\left[y_{0}, y_{0}\right]_{n}\right) & =\left[A_{N-n}^{\star} y_{0}, A_{N-n}^{\star} y_{0}\right]_{n+1}-\left[y_{0}, y_{0}\right]_{n} \\
& =\left(\left(A_{N-n} V_{n+1} A_{N-n}^{\star}-V_{n}\right) y_{0}, y_{0}\right) .
\end{aligned}
$$

We shall assume the following condition to be satisfied for the metrics (3.4) and equation (1.1):

(C) There exists a non-increasing sequence $\left\{\varepsilon_{n}\right\}_{n \in \mathbb{Z}_{+}}$of positive numbers such that

$$
\Delta_{(1.1)}\left(\left[y_{0}, y_{0}\right]_{n}\right) \geq \varepsilon_{n}\left\|y_{0}\right\|^{2} \quad \forall n \in \mathbb{Z}_{+}, y_{0} \in \mathcal{H},
$$

that is,

$$
A_{n}^{\star} V_{n+1} A_{n}-V_{n} \geq \varepsilon_{n} I \quad \forall n \in \mathbb{Z}_{+} .
$$

As a consequence of $(\mathrm{C})$ we obtain

$$
\begin{aligned}
{\left[U(n+1) y_{0}, U(n+1) y_{0}\right]_{n+1}-\left[U\left(n_{0}\right) y_{0}, U\left(n_{0}\right) y_{0}\right]_{n_{0}} } & \\
& \geq \sum_{k=n_{0}}^{n} \varepsilon_{k}\left\|U(k) y_{0}\right\|^{2} \quad \forall y_{0} \in \mathcal{H}, n \geq n_{0} .
\end{aligned}
$$

Consider the following bicones, connected with the metrics (3.4) and the evolution operator of (1.1):

$$
C_{n}^{-}=\left\{y_{0} \in \mathcal{H}:\left[U(n) y_{0}, U(n) y_{0}\right]_{n} \leq 0\right\} \quad\left(n \in \mathbb{Z}_{+}\right) .
$$

Using this definition and (3.7) it is easy to show that

$$
C_{n+1}^{-} \subseteq C_{n}^{-} \text {. }
$$

We set

$$
C_{\infty}^{-}=\bigcap_{n \in \mathbb{Z}_{+}} C_{n}^{-} .
$$

Lemma 3.1. Assume that, besides conditions (A)-(C), the condition

$$
\Delta_{(3.2)}\left(\left[y_{0}, y_{0}\right]_{k}\right) \geq 0 \quad \forall k \in\{0,1, \ldots, N\}, y_{0} \in \mathcal{H}
$$

is satisfied, that is,

$$
A_{N-k} V_{k+1} A_{N-k}^{\star} \geq V_{k} \quad \forall k \in\{0,1, \ldots, N\}, N \in \mathbb{N} .
$$


Then the set $C_{\infty}^{-}$, defined by (3.10), contains a maximal non-positive subspace $L_{\infty}^{-}$of the Krein space $\left(\mathcal{H}, V_{0}\right)$.

Proof. In view of (C), (3.11) with $N=n-1$ and (3.3), we have $[U(n) y, U(n) y]_{n} \geq[y, y]_{0}, \quad\left[U^{\star}(n) y, U^{\star}(n) y\right]_{n} \geq[y, y]_{0} \quad \forall y \in \mathcal{H}, n \in \mathbb{N}$. This means that each $U(n)$ is a $J$-biexpansive operator. Let $M_{n}^{-}$be the set of maximal subspaces of the bicone $C_{n}^{-}$, defined by (3.8), and $\mathcal{M}_{n}^{-}$be the set of angular operators $K^{-}$in the Krein space $\left(\mathcal{H}, V_{0}\right)$, corresponding to the subspaces $L^{-} \in M_{n}^{-}$. By Theorem 2.2, each $\mathcal{M}_{n}^{-}$is compact with respect to the weak operator topology in $\mathcal{L}\left(\mathcal{H}_{0}^{-}, \mathcal{H}_{0}^{+}\right)$, where $\mathcal{H}_{0}^{-}$and $\mathcal{H}_{0}^{+}$are the components of the canonical decomposition of the Krein space $\left(\mathcal{H}, V_{0}\right)$. At the same time, (3.9) implies that $\mathcal{M}_{n+1}^{-} \subseteq \mathcal{M}_{n}^{-}$. Thus $\bigcap_{n \in \mathbb{N}} \mathcal{M}_{n}^{-}$is non-empty. Hence a subspace $L_{\infty}^{-} \in \bigcap_{n \in \mathbb{N}} M_{n}^{-}$is the desired maximal nonpositive subspace of the Krein space $\left(\mathcal{H}, V_{0}\right)$ contained in $C_{\infty}^{-}$.

Let $\left\{\varepsilon_{n}\right\}_{n \in \mathbb{Z}_{+}}$be as in condition $(\mathrm{C})$ and $L_{2, \varepsilon}\left(\mathbb{Z}_{+}, \mathcal{H}\right)$ be the Hilbert space of sequences $\bar{y}=\left\{y_{n}\right\}_{n \in \mathbb{Z}_{+}}$of vectors in $\mathcal{H}$ satisfying the condition

$$
\sum_{n=1}^{\infty} \varepsilon_{n}\left\|y_{n}\right\|^{2}<\infty
$$

with the inner product

$$
(\bar{y}, \bar{z})_{\varepsilon}=\sum_{n=1}^{\infty} \varepsilon_{n}\left(y_{n}, z_{n}\right) .
$$

Denote by $\mathcal{N}$ the set of solutions of (1.1) which belong to $L_{2, \varepsilon}\left(\mathbb{Z}_{+}, \mathcal{H}\right)$, i.e.,

$$
\mathcal{N}=\left\{\bar{y}=\left\{y_{n}\right\}_{n \in \mathbb{Z}_{+}} \in L_{2, \varepsilon}\left(\mathbb{Z}_{+}, \mathcal{H}\right) \mid y_{n+1}=A_{n} y_{n} \forall n \in \mathbb{Z}_{+}\right\} .
$$

Let $\mathcal{N}_{0}$ be the "slice" of $\mathcal{N}$ at time $n=0$, i.e.

$$
\mathcal{N}_{0}=\left\{y_{0} \mid\left(y_{0}, y_{1}, \ldots, y_{n}, \ldots\right) \in \mathcal{N}\right\} .
$$

We now turn to the main result of the present paper.

Theorem 3.1. Assume that besides conditions (A)-(C), the condition

$$
\sum_{n=0}^{\infty} \varepsilon_{n}=+\infty
$$

is satisfied, where $\left\{\varepsilon_{n}\right\}_{n \in \mathbb{Z}_{+}}$is as in (C). Then:

(i) The set $C_{\infty}^{-}$is a closed subspace of $\mathcal{H}$, and

$$
C_{\infty}^{-}=\mathcal{N}_{0}
$$

(ii) For any $y_{0} \in C_{\infty}^{-}$the solution $y_{n}=U(n) y_{0}$ of (1.1) satisfies

$$
\sum_{k=n+1}^{\infty} \varepsilon_{k}\left\|y_{k}\right\|^{2} \leq I\left(y_{0}\right) \prod_{k=0}^{n}\left(1+\varepsilon_{k}\right)^{-1},
$$


where

$$
I\left(y_{0}\right)=\sum_{k=1}^{\infty} \varepsilon_{k}\left\|y_{k}\right\|^{2}
$$

(iii) For any $y_{0} \notin C_{\infty}^{-}$there exists $C\left(y_{0}\right)>0$ such that the solution $y_{n}=U(n) y_{0}$ of (1.1) satisfies

$$
\left\|y_{n}\right\|^{2} \geq C\left(y_{0}\right) \prod_{k=0}^{n}\left(1+\varepsilon_{k}\right)
$$

(iv) If, in addition, condition (3.11) is satisfied, then $C_{\infty}^{-}$is a maximal non-positive subspace of the Krein space $\left(\mathcal{H}, V_{0}\right)$.

Proof. First of all, let us prove (iii). Assume that $y_{0} \notin C_{\infty}^{-}$. Then, in view of (3.8)-(3.10), there exists $n_{0} \in \mathbb{Z}_{+}$such that

$$
\left[U\left(n_{0}\right) y_{0}, U\left(n_{0}\right) y_{0}\right]_{n_{0}}>0 \text {. }
$$

Using condition (B), we obtain from (3.7) the following inequality:

$$
\begin{aligned}
\left\|U(n+1) y_{0}\right\|^{2} \geq & {\left[U\left(n_{0}\right) y_{0}, U\left(n_{0}\right) y_{0}\right]_{n_{0}} } \\
& +\sum_{k=n_{0}}^{n} \varepsilon_{k}\left\|U(k) y_{0}\right\|^{2} \quad \forall n \geq n_{0},
\end{aligned}
$$

which can be rewritten in the form

$$
Y_{n+1}-Y_{n} \geq \varepsilon_{n+1} Y_{n} \quad\left(n \geq n_{0}\right)
$$

where

$$
Y_{n}=\left[U\left(n_{0}\right) y_{0}, U\left(n_{0}\right) y_{0}\right]_{n_{0}}+\sum_{k=n_{0}}^{n} \varepsilon_{k}\left\|U(k) y_{0}\right\|^{2} .
$$

Inequalities (3.19) and (3.21) imply that

$$
Y_{n} \geq\left[U\left(n_{0}\right) y_{0}, U\left(n_{0}\right) y_{0}\right]_{n_{0}} \prod_{k=n_{0}}^{n}\left(1+\varepsilon_{k+1}\right) \quad\left(n \geq n_{0}\right) .
$$

Taking into account (3.19), (3.20) and (3.22), we obtain from the last inequality the desired estimate (3.18) with some positive constant $C\left(y_{0}\right)$.

To prove (i), observe that (3.18) and (3.14) imply that, if $y_{0} \notin C_{\infty}^{-}$, the solution $y_{n}=U(n) y_{0}\left(n \in \mathbb{Z}_{+}\right)$of $(1.1)$ does not belong to $L_{2, \varepsilon}\left(\mathbb{Z}_{+}\right)$. This means that

$$
\mathcal{N}_{0} \subseteq C_{\infty}^{-}
$$

Conversely, assume that $y_{0} \in C_{\infty}^{-}$. Then, by (3.8) and (3.9),

$$
\left[U(n) y_{0}, U(n) y_{0}\right]_{n} \leq 0 \quad \forall n \in \mathbb{Z}_{+} .
$$


This fact and (3.7) imply that

$$
\sum_{k=n_{0}}^{n} \varepsilon_{k}\left\|U(k) y_{0}\right\|^{2} \leq\left[U\left(n_{0}\right) y_{0}, U\left(n_{0}\right) y_{0}\right]_{n_{0}} \quad \forall n \geq n_{0},
$$

hence the solution $y_{n}=U(n) y_{0}\left(n \in \mathbb{Z}_{+}\right)$of (1.1) belongs to $L_{2, \varepsilon}\left(\mathbb{Z}_{+}\right)$, i.e., $y_{0} \in \mathcal{N}_{0}$. So, we have proved (3.15). Since $C_{\infty}^{-}$is closed in $\mathcal{H}$ as the intersection of the closed sets $C_{n}^{-}$, and the set $\mathcal{N}_{0}$ is linear (by (3.12), (3.13)), we have proved (i).

To prove (ii), assume, as above, that $y_{0} \in C_{\infty}^{-}$. Let $n \rightarrow \infty$ in (3.24) and then substitute $n$ for $n_{0}$ to obtain, in view of (B),

$$
\sum_{k=n+1}^{\infty} \varepsilon_{k}\left\|U(k) y_{0}\right\|^{2} \leq\left\|U(n) y_{0}\right\|^{2} \quad \forall n \in \mathbb{Z}_{+} .
$$

Define

$$
Z_{n}=\sum_{k=n+1}^{\infty} \varepsilon_{k}\left\|U(k) y_{0}\right\|^{2} .
$$

Then the last inequality can be rewritten in the form

$$
Z_{n+1} \leq\left(1+\varepsilon_{n+1}\right)^{-1} Z_{n} \quad \forall n \in \mathbb{Z}_{+} .
$$

This estimate and (3.25) imply the desired estimate (3.16).

Assertion (iv) follows from the obvious inclusion $C_{\infty}^{-} \subseteq C_{0}^{-}$and Lemma 3.1.

REMARK 3.1. In [B-G2] an irreversible dynamical system similar to (1.1) was considered under condition (C) with $\varepsilon_{n}=$ const. But the results obtained there differ from those of Theorem 3.1. In particular, in [B-G2] the so-called inertia theorem was proved (Theorem 2.5). It asserts that the operator $\zeta I-T$ is left-invertible for any $\zeta \in \mathbb{T}$, where $T$ is the operator defined in $L_{2}\left(\mathbb{Z}_{+}, \mathcal{H}\right)$ by the block matrix

$$
\left\{\delta_{i, j+1} A_{j}\right\}_{i, j \in \mathbb{Z}_{+}}
$$

and, furthermore, a formula for the dimension of $\operatorname{ker}(\zeta I-T)^{\star}$ is valid in terms of the operators $A_{n}$ and $V_{n}$ (see formula (2.6) of [B-G2]). On the other hand, Theorem 3.1 of the present paper gives information about the asymptotic behavior of sequences from the subspace $\operatorname{ker}(I-T)$, which coincides with the subspace $\mathcal{N}$ defined by (3.12). Theorem 3.1(iv) also gives information about the dimension of the latter subspace. Furthermore, Theorem 3.1 states that all those solutions of (1.1) which do not belong to $\mathcal{N}$ grow to infinity with a certain rate.

The following example shows that condition (3.11) cannot be eliminated in assertion (iv) of the previous theorem. 
EXAMPLE 3.1. Let

$$
\mathcal{H}=\mathcal{H}^{+} \oplus \mathcal{H}^{-}
$$

where

$$
\begin{gathered}
\mathcal{H}^{+}=\operatorname{cl}\left(\operatorname{span}\left(\left\{e_{i}\right\}_{i=1}^{\infty}\right)\right), \\
\left(e_{i}, e_{j}\right)=\delta_{i, j}, i, j=1,2, \ldots, \quad \mathcal{H}^{-}=\operatorname{span}\left(\left\{e_{-}\right\}\right),\left(e_{-}, e_{-}\right)=1 .
\end{gathered}
$$

Define an indefinite metric in $\mathcal{H}$ by $[x, y]=(V x, y)$, where $V=P_{+}-P_{-}$ and $P_{+}, P_{-}$are the orthogonal projections onto $\mathcal{H}^{+}$and $\mathcal{H}^{-}$, respectively. Consider the operator $U=U(\lambda)$ acting in $\mathcal{H}$ and having the following block matrix representation with respect to the splitting (3.27):

$$
U=\left(U_{i, j}\right)_{i, j=1}^{2},
$$

where

$$
\begin{gathered}
U_{1,1} e_{k}=e_{k+1}, k \in \mathbb{N}, \quad U_{1,2} e_{-}=e_{1}, \\
U_{2,1}=0, \quad U_{2,2}=\lambda I, \quad \lambda \in \mathbb{C},|\lambda| \leq \sqrt{2} .
\end{gathered}
$$

Then $U=U(\lambda)$ is a bounded left-invertible operator, and $[U x, U x]-[x, x]=$ $|\beta|^{2}\left(2-|\lambda|^{2}\right) \geq 0$ for any $x=\sum_{k=1}^{\infty} \alpha_{k} e_{k}+\beta e_{-} \in \mathcal{H}$, that is, $U$ is a $J$-expansion. Let us calculate $\mathcal{G}_{U(\lambda)}^{-}$. A simple criterion for $K^{-} \in \mathcal{G}_{U(\lambda)}^{-}$is the following (see [Kh4, proof of Theorem 2.1]):

$$
\begin{aligned}
\left(K^{-}\right)^{\star}\left(U_{1,1}^{\star} U_{1,1}-U_{2,1}^{\star} U_{2,1}\right) K^{-}+\left(K^{-}\right)^{\star}\left(U_{1,1}^{\star} U_{1,2}-U_{2,1}^{\star} U_{2,2}\right) & \\
& +\left(U_{1,2}^{\star} U_{1,1}-U_{2,2}^{\star} U_{2,1}\right) K^{-}+\left(U_{1,2}^{\star} U_{1,2}-U_{2,2}^{\star} U_{2,2}\right) \leq 0 .
\end{aligned}
$$

In our case

$$
U_{2,1}=U_{1,1}^{\star} U_{1,2}=0, \quad U_{1,1}^{\star} U_{1,1}=U_{1,2}^{\star} U_{1,2}=I, \quad U_{2,2}^{\star} U_{2,2}=|\lambda|^{2} I,
$$

and (3.30) becomes

$$
\left\|K^{-}\right\|^{2}+1-|\lambda|^{2} \leq 0
$$

This shows that

$$
\begin{array}{ll}
\mathcal{G}_{U(\lambda)}^{-}=\emptyset & \text { for }|\lambda|<1, \\
\mathcal{G}_{U(\lambda)}^{-} \neq \emptyset & \text { for } 1 \leq|\lambda| \leq \sqrt{2} .
\end{array}
$$

Consider the operator $A=c U,|c|>1$, and the autonomous difference equation

$$
y_{n+1}=A y_{n} .
$$

Take any $y_{0}=\sum_{k=1}^{\infty} \alpha_{k} e_{k}+\beta e_{-} \in \mathcal{H}$. Then

$\left[A y_{0}, A y_{0}\right]-\left[y_{0}, y_{0}\right]=\left(|c|^{2}-1\right) \sum_{k=1}^{\infty}\left|\alpha_{k}\right|^{2}+|\beta|^{2}\left(1+|c|^{2}\left(1-|\lambda|^{2}\right)\right) \geq d\left\|y_{0}\right\|^{2}$,

where $d=\min \left\{|c|^{2}-1,1+|c|^{2}\left(1-|\lambda|^{2}\right)\right\}$. Evidently, $d>0$ for $|\lambda| \leq 1$ and any $c$ with $|c|>1$, and also for $1<|\lambda| \leq \sqrt{2}$ and $1<|c|<1 / \sqrt{|\lambda|^{2}-1}$, i.e., 
in these two cases condition (C) is satisfied for equation (3.33) with $\varepsilon_{n}=d$. It is clear that also conditions (A), (B) are satisfied for $[x, y]_{n}=[x, y]$. On the other hand, in view of (2.5) and (3.31), $C_{n}^{-}=\{0\}$ for each $n \in \mathbb{N}$, hence $C_{\infty}^{-}=\{0\}$ for all $\lambda \in \mathbb{C}$ with $|\lambda|<1$ and

$$
\operatorname{dim}\left(C_{\infty}^{-}\right)=1 \quad \forall \lambda \in \mathbb{C}, 1 \leq|\lambda| \leq \sqrt{2} .
$$

Thus for $|\lambda|<1$ Theorem 3.1 implies that for any $0 \neq y_{0} \in \mathcal{H}$ the solution $y_{n}=U(n) y_{0}$ of (3.33) tends to infinity exponentially as $n \rightarrow \infty$. This means that the stable subspace of (3.33) is $\{0\}$, which is evidently not a maximal subspace in $C_{0}^{-}$. So, for $|\lambda|<1$ assertion (iv) of Theorem 3.1 is not valid.

But if $1 \leq|\lambda| \leq \sqrt{2}$ we have the following picture in view of (3.32): by Theorem 3.1(ii), (iii), for any $y_{0}$ in the one-dimensional subspace $C_{\infty}^{-}$ the solution $y_{n}=U(n) y_{0}$ of (3.33) tends to zero exponentially as $n \rightarrow \infty$, and for any $0 \neq y_{0} \in \mathcal{H} \backslash C_{\infty}^{-}$it tends to infinity exponentially as $n \rightarrow \infty$. Observe that, in view of (3.34), in this case assertion (iv) of Theorem 3.1 is valid.

Let us show that condition (3.11) holds for neither $|\lambda|<1$ nor $1 \leq|\lambda|$ $\leq \sqrt{2}$. Indeed, for $y_{0} \in \mathcal{H}^{+}$,

$$
\left[U^{\star} y_{0}, U^{\star} y_{0}\right]-\left[y_{0}, y_{0}\right]=-2 .
$$

REMARK 3.2. A simple version (with $\lambda=0$ ) of Example 3.1 has been considered in [B-G2] as an example of equation (1.1) which satisfies condition (C), but $\zeta I-T$ is not right-invertible for any $\zeta \in \mathbb{T}$, where $T$ is defined in $L_{2}\left(\mathbb{Z}_{+}, \mathcal{H}\right)$ by the block matrix (3.26).

REMARK 3.3. The case $1 \leq|\lambda| \leq \sqrt{2}$ of Example 3.1 shows that condition (3.11) is not necessary for assertion (iv) of Theorem 3.1 to be valid.

REMARK 3.4. It would be interesting to construct an example analogous to the case $|\lambda|<1$ of Example 3.1 for an evolution equation with continuous time:

$$
\frac{d x}{d t}=A(t) x
$$

Observe that there is little chance to construct such an example for the autonomous equation $(A(t)=A=$ const). If we have a strict plus-operator $A$ with $\mathcal{G}_{A}^{-}=\emptyset$ and try to extend the discrete semigroup $\left\{A^{n}\right\}_{n \in \mathbb{Z}_{+}}$to a semigroup with continuous time $t \in[0, \infty)$, we see that the operator $A^{t}$ does not exist in the sense of Dunford for $t \notin \mathbb{Z}_{+}$by Proposition 2.1. But it would be interesting to construct such an example for a non-autonomous dynamical system of the type (3.35).

Example 3.2. In a Hilbert space $\mathcal{H}$ consider the operator $A$ defined in Example 3.1 with $\lambda=0$, that is, $A=c U(0)(c>1)$, where $U(\lambda)$ is the operator defined by the block matrix representation (3.28), (3.29). Consider 
the Hilbert space $\widetilde{\mathcal{H}}=\mathcal{H} \oplus \mathbb{C}$ and denote by $(\cdot, \cdot)_{\widetilde{\mathcal{H}}}$ the inner product in it. Define an indefinite inner product in $\widetilde{\mathcal{H}}$ by $[x, y]_{\widetilde{\mathcal{H}}}=(\widetilde{V} x, y)_{\widetilde{\mathcal{H}}}$, where $\widetilde{V}=V \oplus(-I)$ and $V$ is the operator from Example 3.1, which defines the indefinite metric in $\mathcal{H}$. Also consider the operator $\widetilde{A}=A \oplus \theta I$ with $\theta \in(0,1)$ and the corresponding autonomous difference equation in $\widetilde{\mathcal{H}}$ :

$$
y_{n+1}=\widetilde{A} y_{n} .
$$

It is easy to check that for some $\varepsilon>0$,

$$
\Delta_{(3.36)}\left(\left[y_{0}, y_{0}\right]_{\tilde{\mathcal{H}}}\right) \geq \varepsilon\left(y_{0}, y_{0}\right)_{\tilde{\mathcal{H}}} \quad \forall n \in \mathbb{Z}_{+}, y_{0} \in \widetilde{\mathcal{H}}
$$

Furthermore, $d_{\widetilde{V}}^{-}=2$, because $d_{V}^{-}=1$ in $\mathcal{H}$. On the other hand, in view of the result of Example 3.1, equation (3.36) exhibits the exponential dichotomy with a one-dimensional stable subspace. So, in the constructed example the dichotomy is not compatible with the signature of the indefinite metric.

\section{References}

[Az-I] T. Ya. Azizov and I. S. Ǐohvidov, Linear Operators in Spaces with an Indefinite Metric, Wiley, Chichester, 1989.

[A-M-Z] B. Aulbach, Nguyen Van Minh and P. P. Zabreiko, The concept of spectral dichotomy for linear difference equations, J. Math. Anal. Appl. 185 (1994), 275-287.

[A-S] B. Aulbach and S. Siegmund, The dichotomy spectrum for noninvertible systems of linear difference equations, J. Difference Equations Appl. 7 (2001), 895-913.

[B-P] A. G. Baskakov and A. I. Pastukhov, Spectral analysis of a weighted shift operator with unbounded operator coefficients, Siberian Math. J. 42 (2001), 10261035 .

[B-G1] A. Ben-Artzi and I. Gohberg, Inertia theorems for block weighted shifts and applications, in: Oper. Theory Adv. Appl. 56, Birkhäuser, Basel, 1992, 120-152.

[B-G2] - - - Inertia theorems for operator pencils and applications, Integral Equations Operator Theory 21 (1995), 270-318.

[B-G3] - - - Inertia theorems for non-stationary discrete systems and dichotomy, Linear Algebra Appl. 120 (1989), 95-138.

[B-G4] - - - Fredholm properties of band matrices and dichotomy, in: Oper. Theory Adv. Appl. 32, Birkhäuser, Basel, 1988, 37-52.

[B-G5] - - -, Dichotomy, discrete Bohl exponents, and spectrum of block weighted shifts, Integral Equations Operator Theory 14 (1991), 613-677.

[Kh1] V. Khatskevich, Some global properties of fractional-linear transformations, in: Operator Theory Adv. Appl. 73, Birkhäuser, Basel, 1994, 355-361.

[Kh2] - Characteristic spectral properties of focusing operators, Akad. Nauk Armyan. SSR Dokl. 79 (1984), 102-105 (in Russian).

[Kh3] - Symmetry of the properties of a plus-operator and its conjugate, in: Functional Analysis, No. 14, Ul'yanovsk. Gos. Ped. Inst., Ul'yanovsk, 1980, 177-186 (in Russian).

[Kh4] -, Generalized fractional linear transformations: convexity and compactness of the image and the pre-image; applications, Studia Math. 137 (1999), 169-175. 
[Kh-Sh] V. Khatskevich and V. Shulman, Operator fractional-linear transformations: convexity and compactness of image; applications, ibid. 116 (1995), 189-195.

[Kh-Z1] V. Khatskevich and L. Zelenko, Indefinite metrics and dichotomy of solutions of linear differential equations in Hilbert spaces, Chinese J. Math. 24 (1996), 99-112.

[Kh-Z2] -, 一, Bistrict plus-operators in Krein spaces and dichotomous behavior of irreversible dynamical systems, in: Oper. Theory Adv. Appl. 118, Birkhäuser, Basel, 2000, 191-203.

[Kr] M. G. Krein, On a new application of the fixed point principle to operator theory in spaces with indefinite metric, Soviet Math. Dokl. 5 (1964), 224-228.

[Mz] A. D. Maizel, On stability of solutions to systems of differential equations, Ural. Politekhn. Inst. Trudy 51 (1954), 20-50 (in Russian).

[Ms-Sh] J. L. Massera and J. J. Schäffer, Linear Differential Equations and Function Spaces, Academic Press, New York, 1966.

[Shm] Yu. L. Shmul'yan, On divisibility in the class of plus-operators, Mat. Zametki 74 (1967), 516-525 (in Russian).

[Z] L. Zelenko, On the manifold of square integrable solutions of nonlinear systems of differential equations, in: Differential Equations and Theory of Functions, No. 6, Saratov Univ., Saratov, 1984, 19-26 (in Russian).

Department of Mathematics

ORT Braude Academic College

P.O. Box 78, 21982 Karmiel, Israel

E-mail: victor_kh@hotmail.com
Department of Mathematics University of Haifa 31905 Haifa, Israel E-mail: zelenko@math.haifa.ac.il

Received August 13, 2003

Revised version October 4, 2006 